\title{
HE staff's attitudes and expectations about their role in induction activities
}

\section{Camila Devis-Rozental}

Bournemouth University, UK

\section{Susanne Clarke}

Bournemouth University, UK

\section{Abstract}

The views of higher education staff regarding their role in the induction period have not been fully explored. Yet this transition to university is a complex period for students. In the UK, many students who are going to university leave home, some for the first time. As they arrive they have to deal with many new and sometimes difficult situations they may not have encountered before. During the induction period students come across many staff within the university and these interactions are vital to support students in developing a sense of belonging within the university community. This small-scale project sought to evaluate the current provision for the induction process in a UK university to identify areas for improvement, by seeking views regarding the induction activities from staff within a UK university. Findings from a staff survey with 58 participants suggest opportunities to improve practice. The main areas identified were a need for better communication between teams, and effective training and support for staff to understand the issues students may face and the type of support they will need. Additionally, the need to develop a more unifying understanding of every member of the university as an active participant within the induction process was highlighted.

Keywords: student experience; transitions; HE staff; HE staff experience. 


\section{Introduction}

This paper presents the findings from a research project undertaken in a UK university exploring the views of staff on the transition of students to university. This mixed methods research project was carried out through a survey conducted with 58 academic and professional members of staff and sought to investigate their views concerning the induction process, the role they played within it, and their expectations of the students starting university. The aim of the study was to evaluate current practice and present a series of recommendations for practice improvement.

\section{Background and literature}

Although literature around students' transition to university is evident (Yorke and Longden, $2004 ; 2008$ ), literature on university staff's (both academic and professional) expectations of students when they first arrive at university is scarce (Hassel and Ridout, 2018). The available literature concentrates on academic staff. Fraser and Killen (2003) explore the expectations of staff and students, concluding that these tend to be very different, for example regarding attendance at lectures and self-motivation. However, their work concentrates on lecturers rather than taking on an all-staff approach. Hassel and Ridout (2018) found that it is key to ensure that student and staff expectations concur, since disparity between each group's expectations can result in 'communication breakdown' between staff and students (p.11). This way of working together between academic and professional members of staff ensures students are supported more effectively and is therefore very important within the first-year student experience (Wojcieszek, 2014), and arguably within the induction period as students are settling in.

The transition to university can be challenging in many areas such as developing academic skills and friendships (Perry et al., 2001), as well as pedagogical expectations (Jones, 2018). Tinto (1999) argues that students leave their programmes during their first year because they lack academic skills, coupled with other important aspects such as finance issues, isolation, and a lack of social integration. Ensuring students get the appropriate information and support during their induction could potentially reduce this. Although not a new idea, it seems that in 
the past few years there has been an increase in students entering higher education with multifaceted issues related to their mental health, lacking the academic skills required at this level or with unrealistic expectations of what university life will be like, and this is of concern within the sector (Houghton and Anderson, 2017). Many students enter university without an understanding of the requirements needed for university. This can be in areas related to their wellbeing such as self-care or time management, but also relate to the academic skills required such as academic writing, referencing, or even understanding the marking criterion (Devis-Rozental, 2018; 2020; Jones, 2018).

Additionally, in many cases they will have to get to know a new city, develop new friendships and, for those moving from another city and living away from home, this can be extremely difficult (Devis-Rozental, 2018). These many new ways of being can exacerbate the barriers and obstacles they already have, which can diminish their ability to learn effectively (Rozental-Devis, 2020).

A survey by HEPI and the HEA (Seldon and Martin, 2018) found that students are 'doing less well than 20 to 24 year-olds from the general population on four measures - life satisfaction; whether life feels worthwhile; happiness; and anxiety'(p.10). Thus, it is important to consider these areas when supporting students as they transition into university.

Seldon and Martin (2018) argue that staff in schools and colleges should take the time to support students in developing the academic and personal skills they will need once they start university: areas such as preparing students to manage their finances and schedule their time, as well as areas which affect students' holistic wellbeing. Still, a focus on exams and results keeps teachers from addressing issues related to preparing students for university (van Rooij, 2018).

There are few studies exploring the views of academic and professional staff within universities and the role they play in this transition. Jones (2013) argues that 'a cultural change may be required for all staff within $\mathrm{HE}$ to view themselves as stakeholders in supporting and enhancing the student experience' (p.165). Consequently, a whole institution approach to supporting students in this transition must be sought (Clark et al., 2015). This is 
the case even though the roles that academic and professional members of staff will have during this period may differ, because what is important is to have a positive attitude, a proactive approach, and consistency from those involved in the induction in order to be able to support and signpost students to the right type of support (Jones, 2013).

Research shows that students develop their socio-emotional intelligence during their time at university by interacting with those around them (Devis-Rozental and Farquharson, 2020). Therefore, ensuring staff are effective role models and demonstrate prosocial attributes and behaviours from induction onwards is an important aspect that must be considered.

Nevertheless, with an emphasis on the student experience, it may be that the views of those delivering this induction and supporting the transition have not been fully considered. Therefore, this paper aims to bridge this gap by exploring the views of academic and professional staff regarding this transition.

\section{Context}

There has been a marked improvement in how our university supports students transitioning to university, from the students' point of view. For instance, we now have a dedicated induction and transition team and a steering group in charge of general induction activities and practicalities. Data collected from a survey of students during induction in 2017-18 and 2018-19 identified areas of good practice that have improved the students' experiences throughout this transition, such as the increased availability of student accommodation, the success of the activities planned for students, and the use of the residential life team.

The report written to address the survey findings identified a series of recommendations related to logistics, operations communications, and IT support, in order to improve or streamline our support for students rather than change current provision. Feedback from students was mostly positive, giving the university's provision a sound framework from which to continue developing. 
A survey carried out within the university to discover how academics understand their role in supporting their students' health and wellbeing found that most academics see this as part of their practice, even during induction. It also found that there needs to be more clarity regarding boundaries and a need to support staff to keep up to date about signposting.

In an attempt to involve all staff, both academic and professional, this research project carried out a survey where all university employees could answer a series of questions regarding the type of support that students may need in their transition from school to university as well as their role within this period.

\section{Methodology}

Following the university's ethical protocol, a survey was distributed through the university blogs as well as through the Heads of Education in each faculty, the internal communications team, and a twitter message during the academic year 2018-2019. One of the aims of the survey was to explore the views of all staff (academic and professional) regarding their involvement in the induction process at this university.

Measuring staff opinions can be challenging through a survey if it merely looks at quantitative data. As these responses are based on the participants' lived experiences, we chose to conduct a mixed methods study. The survey included qualitative data through open ended questions, supplemented by some relevant quantitative data. These data were gathered in parallel and were integrated within the analysis phase with the aim to enrich our understanding (Greene, 2007). Groves et al. (2004) argue that 'a survey is a systematic method for gathering information from (a sample of) entities for the purpose of constructing quantitative descriptors of the attributes of the large population of which the entities are members' (p.2).

The reason for choosing a survey was to gain information from a larger sample, as the survey was shared with all staff. Additionally, the survey was anonymous as we were keen to elucidate on areas that perhaps in other situations colleagues would not be comfortable 
discussing. There are drawbacks to carrying out surveys. For instance, it may be difficult to clarify questions to participants or a poor response rate. Therefore, when designing a study, it is important to be aware of sample sizes for methodological and ethical reasons and to ensure the data generated is reliable (Faber and Fonseca, 2014). Marshall et al. (2013) assert that qualitative methodologists do not agree on a standard when looking at sample size. They go on to say that sample size may be culturally influenced. Therefore, it may be difficult to ascertain the right number of participants that will give you the data you need. Even though the sample size for this research was small due to poor response, there were themes emerging which allowed us to generalise some of our findings. These concurred with some of the literature explored and raised interesting points for us to continue improving our provision.

Another issue is around a participant's understanding or lack of understanding of the questions, which, without direct contact, cannot be ascertained. We aimed to minimise this by creating very simple questions which were easy to understand. We also included our details in case anyone would like to contact us for further clarification. If we conducted this research again and with more time available, we would have followed up the survey with focus groups or qualitative interviews to gain a more in-depth understanding of the issues identified.

When developing the sampling plan, it was decided that all staff (academic and professional) would be invited to take part in an online survey (about 600). This was an opportunity to gain rich data on their experiences and views around our induction process and their expectations of students during this time. When developing the questions, we looked carefully at language, using words that were consistent and easy to understand and avoiding biased language (Browne and Keeley, 1998).

\section{Participants}

Although from a quantitative point of view we did not have a large response to our call, 58 members of staff completed the survey and provided rich data for us to explore. Of those, $47 \%$ were academics and $44 \%$ professional members of staff. $9 \%$ did not specify their role (see Figure 1). 
Figure 1. Staff who answered the survey.

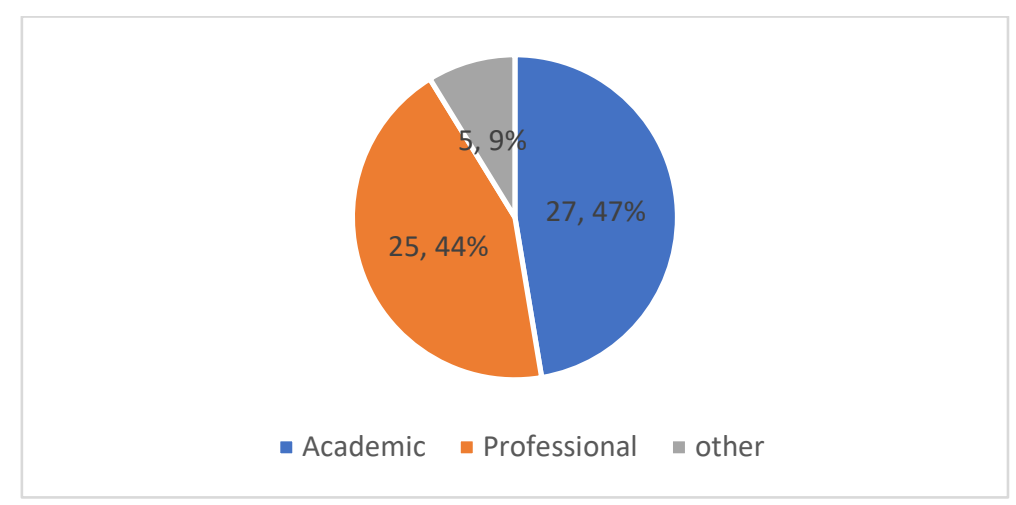

Clearly there was engagement in the survey by academics as well as professional members of staff: 'This is important as everyone should be involved in the induction regardless of their job role at the university' (Devis-Rozental and Barron, 2020, p.97).

\section{Findings and discussion}

This paper sought to find out the expectations of staff regarding the transition period and activities within the university. In order to understand this, it was important to know the type of support participants felt they provided to students. Figure 2 illustrates that most people answering the survey view their role as including opportunities to support students with many aspects, such as emotional issues.

Figure 2. Responsibility within your role.

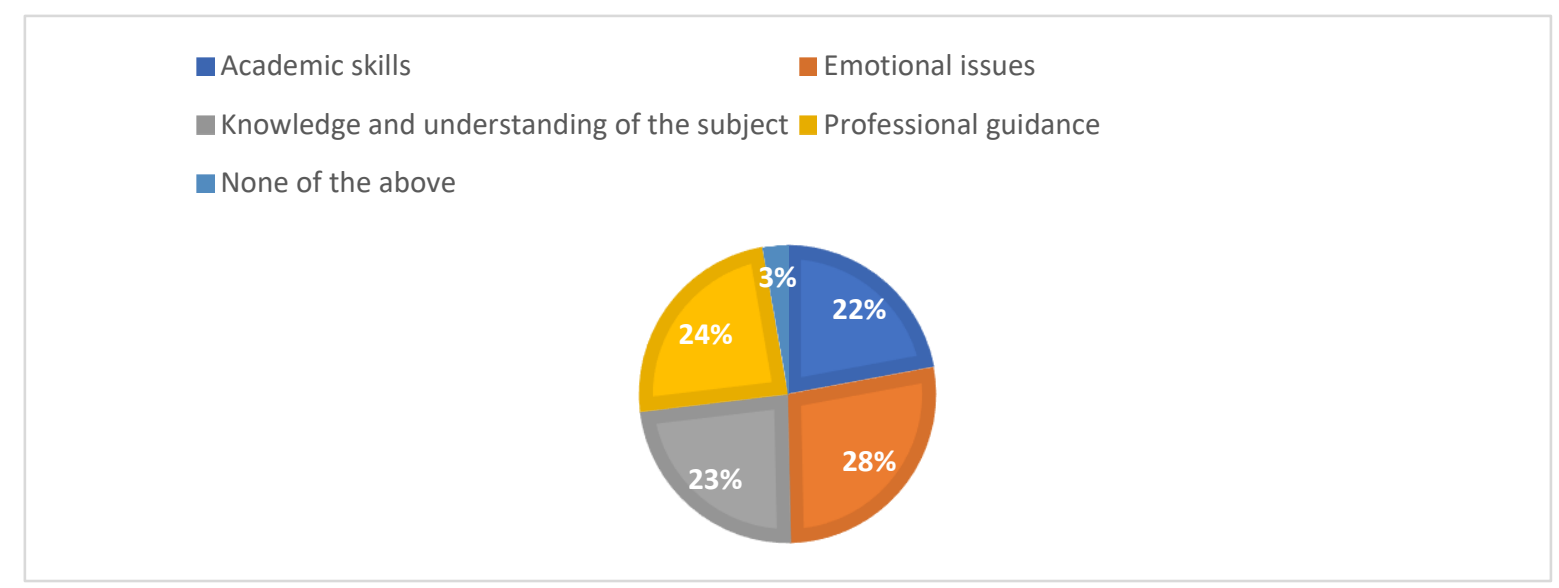


Additionally, participants also view their role as providing students with knowledge of the subject as well as academic skills support. This is a positive find which addresses Tinto's 1999 point regarding students leaving university because they lack the academic skills needed at this level. This is not to say that students will gain all the skills they need during their induction; in fact this would not be productive as it would not be applicable or relevant at this point in their journey. Nevertheless, it would signal its importance and perhaps ease their worries, as highlighted by Devis-Rozental and Barron (2020).

Most academic staff identified that they supported students with emotional issues.

Professional members of staff also felt that emotional issues were something they would be able to support students with. This addresses Seldon and Martin's (2018) points regarding the type of support students should receive during this transition.

Most participants saw their role as one where they were able to support students holistically. This is vital because it means that they take into account the socio-emotional context of learning and developing, something already identified in the literature by Devis-Rozental and Barron (2020) as evident. This was closely followed by the other areas. It is interesting to note that four participants felt that they did not give any type of support to students. There may be a misunderstanding of what support means to students and if it differs from what staff think. Taking into account Jones' (2013) point of a need for a culture shift so that all staff within the university feel they have a part to play in the student experience, this is an area where improvements could be made through further training, for example.

\section{Inductions}

The next section explored the staff's views regarding their involvement within the induction period. Over half of those who answered indicated that they were involved in the process (Devis-Rozental and Barron, 2020). Figure 3 shows that 17 participants did not think they were involved in inductions and four were not sure. 
Figure 3. Involvement in the university induction process.

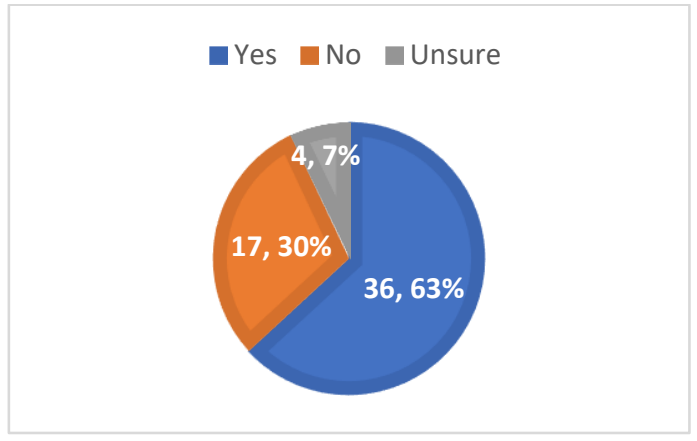

In recent years, the university has emphasised the importance of all staff being involved in the induction process. Still there are some members of staff that clearly do not see themselves as being part of it. Having the essential information, skills, and behaviours that will support students during this transition is important. It does not have to be in depth knowledge of a subject or programme but rather a positive attitude, so students feel they can approach us, and having the right type of information so that we can either support them or send them to the right place (Baer, 2008; Mah and Ifenthaler, 2017; Devis-Rozental and Barron, 2020). There must be a clearer message and support for staff to develop their understanding and knowledge so that they feel part of the induction activities. This in turn will have a positive impact on the student experience.

\section{Student expectations}

When asked what they thought about the expectations of students concerning the induction process, 40 staff participants stated that students sought general information. 13 of those asked stated that students needed (not expected) a warm welcome and to feel that they were part of the university community. There seems to be a disparity in this answer if we consider that in question four, 40 participants mentioned emotional support (pastoral), but in this question only 13 did so. Consequently, this is an area that could be further explored to fully understand the reasons why some people think students may need emotional support, but do not expect it as part of the induction process.

Three participants stated that students did not expect anything or 'not a lot' and four did not know what students would expect, with one participant stating: 'they have no real idea of what 
to "expect" as such' and another one stating 'I'm not sure they expect much at all'. From research we know that students come to university with a multitude of expectations, and it is important to ensure we know what these are and how to meet them if possible (DevisRozental and Barron, 2020).

Most participants mentioned effective communication as something students expected. For instance, one participant stated students expected 'good communication and signposting' whilst others mentioned 'knowledge', 'clear, 'concise', 'accurate' information and advice, with one other stating that students expected 'a font of all knowledge! Definitive answers'. Thus, effective communication and the knowledge of where to find the right type of information were important to them.

\section{Staff expectations}

Regarding the staff's own expectations of commencing students during the induction process, there was a variety of responses, although it is clear that the main expectation was engagement.

Figure 4. Staff expectations of commencing students.

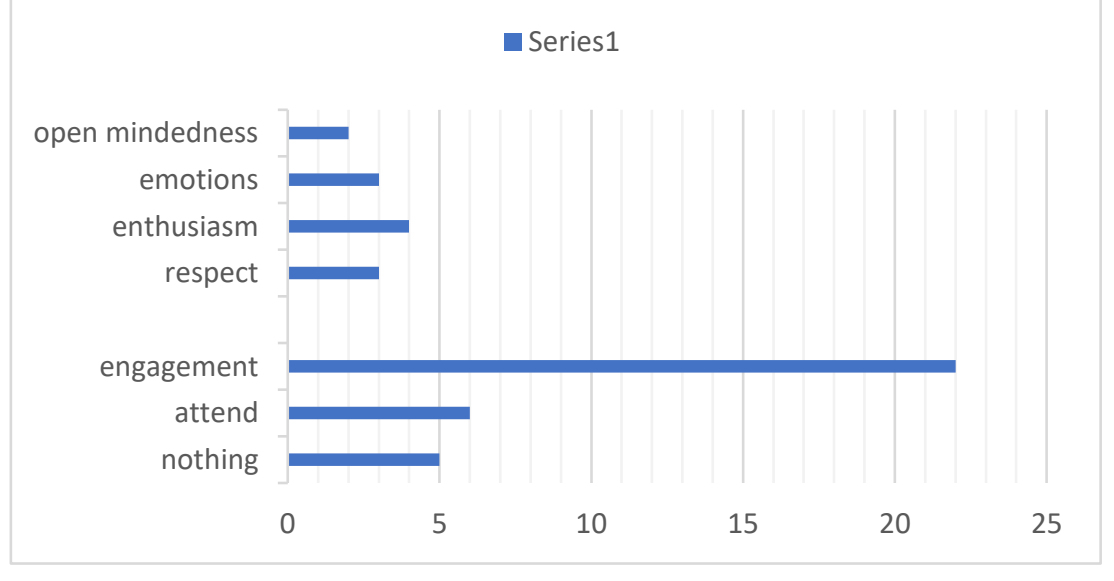

Almost half of the participants expected students to engage with the induction activities by taking part actively. Participants mentioned the importance of resilience and the knowledge that they (students) would have to work hard and engage. Other responses included confidence to speak up if they feel lost, respect, and engagement. Attendance of sessions 
and engagement were also seen as important, although one participant conceded that perhaps we should not expect students to engage at this point as it could be 'scary' to do so. Staff role-modelling prosocial attitudes and behaviours could encourage students to do so, too (Devis-Rozental and Farquharson, 2020).

Clearly most staff have expectations of students and are aware of the needs they may have. There were five participants who expected nothing and six only expected students to turn up. This does not concur with current research which has found that students do have expectations of their university experience and it is important that these expectations match those of staff (Fraser and Killen, 2003; Ridout, 2018). One participant stated 'I expect to bore them senseless with information they are too excited/bored to take in'. Clearly, this is not the right attitude and is something which could impact negatively on the experience of a newlyarrived student (Devis-Rozental and Farquharson, 2020).

Considering the lived experiences of students before they arrive at university is important in order to cater for their needs (Jones, 2018), especially as this is an emotionally charged transition where the majority of students are leaving their family and home life behind, and students will therefore need support in order to settle (Rozental-Devis, 2020). An option to improve practice could be better training for all staff regarding students' expectations, the barriers they face, and issues of accessing university. Staff working at a university should have opportunities to gain knowledge and understanding of how students are currently prepared before they join their university (Seldon and Martin, 2018).

Conversely, and thinking about the specific knowledge students should have before they arrive at university, the idea of having no expectations and an open mind can be beneficial as it may take away added pressure or anxiety for students. For instance, staff could use the tourist metaphor developed by Devis-Rozental (2018) as a framework for ensuring students gain the required knowledge and skills without making assumptions. This metaphor explores the idea of designing 'strategies to provide an environment that positively impacts on students' (Devis-Rozental, 2018, p.104), by seeing them as migrants arriving at our university with no previous knowledge of our customs (policies), currency (marks), language (academic 
writing), laws (plagiarism), geography (campuses), thus avoiding assumptions regarding their knowledge and understanding.

Doing so would enable universities to develop comprehensive induction packages to ensure students gain the basic knowledge and skills they need to thrive at university. As it stands, there is already a benchmark to ascertain that students have the competence to be at university in the way they go through the application and acceptance stages, assuming it works well. So, once they have gone through that and we know they have the minimum requirements, welcoming them with unconditional positive regard, without prejudice or preconceived ideas but with a positive and welcoming attitude can be beneficial to all (DevisRozental, 2018).

\section{Usefulness of the induction process}

The last question asked staff about the current induction and what students get from it.

\section{Figure 5. Student gain from the current induction.}

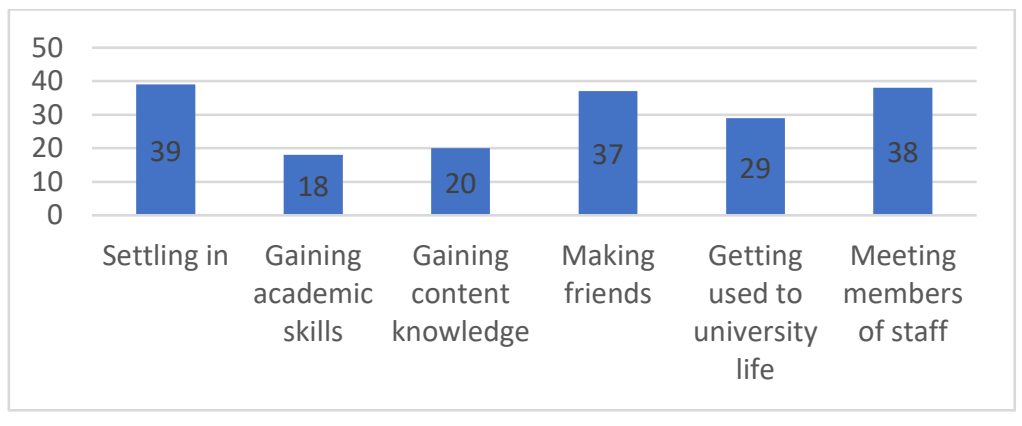

The majority of respondents envisaged the induction activities as an opportunity to settle and to get to know those around them. Developing both a sense of belonging and meaningful relationships at university are key influencers on students' university experiences and can be predictors of success (Devis-Rozental and Clarke, 2020). Consequently, it is vital to ensure that these activities encourage opportunities for social interactions so that students can develop relationships and 'have the time and space to settle in' (Rozental-Devis, 2020 p.79). 
Meehan and Howells (2017) found that the three main things that matter to students as they enter university are the relationships they form with the academic staff, the course they are doing, and a developing sense of belonging. Therefore, ensuring universities enable opportunities for students to do these things during their induction period is important. Ensuring that staff attitudes are positive and engaging and the activities organised are developed with a view to building effective relationships with other students are key areas (Jones, 2013). Additionally, there should be opportunities for staff to interact with the students so that individuals feel part of the community and, in a way, peers in the learning journey (Devis-Rozental, 2018; 2020).

Seldom and Martin (2018) offer other suggestions which could be incorporated even before students arrive on campus, such as having contact with a personal mentor, which they suggest could be a second-year student. In this way, students would be able to ask questions of a peer and perhaps feel less isolated once they arrive on campus. This would address the issues raised in the survey regarding students feeling welcomed and supported.

\section{Analysis: we are all in this together}

The transition to university is a complex time and can be difficult for some students. A report by HEPI (Seldon and Martin, 2018) identified the importance of taking a whole university approach. This is something that is important and sometimes overlooked or difficult to achieve due to silos, and sometimes misunderstandings about everyone's role within the induction process, as found in this research. However, this presents a sound opportunity to provide consistency between teams and different areas of our provision and to ensure students will be well supported as staff are well informed and knowledgeable about how best to support students.

Our university has taken a whole university approach in its central induction activities. Through a steering group, the various stakeholders in different areas of the university have worked collaboratively to ensure all students have the right support and activities. Still, it 
seems that not all staff are aware of this and the important role each of us plays, whatever it is we do.

Therefore, more effective communication channels between teams to ensure every member of staff within the university community understands how their role can impact on the induction activities and the ability of students to settle in, are needed. This is particularly important for those members of staff who did not feel part of the induction process. It does not have to mean that we should all be experts in all aspects of the university. It is more about understanding that our attitude, prosocial behaviours, and willingness to help can make a difference to the student experience.

Information sessions could be put into place to ensure all staff feel they can address any issues or questions regarding this transition, or where to signpost students to. Also it is key to get them to understand the role they can play as culture leaders representing our university and to consider the importance of providing opportunities for developing meaningful relationships, a sense of belonging, and some general knowledge of their programmes; the three areas identified by Meehan and Howells (2017) as important to students.

We are keen to ensure that every member of staff in our university sees themselves as culture leaders, role modelling the socio-emotional and professional behaviours and attitudes that we aim for our students to develop. Research shows that students develop these skills at university from those around them (Meehan and Howells, 2017; Devis-Rozental, 2018; 2020; Devis-Rozental and Farquharson, 2020).

Consequently, an evaluation of the current channels and support for staff, and a stock taking exercise to map the available support to students, both academic and personal, is being undertaken to continue streamlining the communication channels and to provide more clarity and efficiency to both students and staff. This is important because without teams knowing and understanding what other teams can offer, there may be unnecessary duplication of activities which would not be cost effective, or signposting issues which could be frustrating for students. What is more, repetition and duplication can be confusing to students seeking clarity. Additionally, having disparity on the type of support offered to students is unfair to 
those who do not have access to the same level of support for whatever reason. To ensure consistency of approach with clear channels it is vital that all staff can signpost students to the best support for their needs.

Most academic staff are traditionally involved within the transition as they will have the most contact with their students. Still, it is important to acknowledge that non-academic staff also play a key role within these first weeks and their attitude, behaviour, and message to the students must be similar to that of academic staff to provide a consistency of approach. Jones (2013, p.165) argues that these staff 'make an important contribution to the students ... through their interactions'. This should be encouraged with appropriate training and support for all staff so that they feel comfortable taking on these roles. This is possible (Clark et al., 2015) and would also go towards further humanising higher education practice by breaking down a 'them and us' culture between academics and professional staff which sometimes exists at universities and which goes against having a whole university approach (DevisRozental and Clarke, 2020).

\section{Conclusions}

At present, and based on the findings from this research, most staff that answered the survey in this project see induction as a time to give information rather than support. However, considering the emotive nature of these transitions, it is important to be vigilant about students' wellbeing and to support them effectively or signpost them to the right type of support during this time. These skills will probably be centred on practical activities such as budgeting, home arrangements, or making friends rather than academic expectations. Being able to signpost every student to the correct team to seek support with confidence is therefore key.

Overall, this paper found that most colleagues who answered the survey see the induction period as important and feel part of it. However, specific training to ensure staff are able to support students during this transition with consistency may be needed, as some staff are not clear about their role. 
Additionally, it found that there is a need for better communication channels between the different services and departments within the university, to ensure staff are well informed and that students are signposted to the right type of support, as well as to provide a more consistent approach, something highlighted in the literature. This is particularly important as some participants do not feel engaged with students within the induction period as discussed previously within this paper.

Consequently, ensuring staff develop an awareness and understanding of the vital role that each member of our university community plays during the induction period is essential. This is particularly critical since this can be an emotionally charged period for students, and staff's lack of engagement, knowledge, or clear understanding of their impact on students can be detrimental to the student experience and can ultimately result in students leaving.

\section{Limitations and blind spots}

This was a small-scale study and may present some limitations regarding generality; in the future a larger study should be considered. Additionally, further exploration of some of the areas could have been included by carrying out post-survey interviews, for example. Perhaps using the survey as the data collection method was restrictive as we were not able to follow up with specific participants due to their anonymity, especially considering that we were looking at attitudes and opinions, and some responses needed further clarification. This is something that may have been better explored through interviews or focus groups and should be considered in future research studies.

Another limitation was the lack of literature looking at staff attitudes about transition. However, this identified a new gap in the literature to be further researched which this project has aimed to tackle, albeit on a small scale. Consequently, further research in the area is still needed. 
Lastly, the main purpose of this research project was to enrich practice and support current provision in a single university. Looking at different types of provision and what works for them could add richness to this type of research and should be further explored.

\section{References}

Baer, L. D. (2008) 'Misunderstandings about student transitions to university: a slow-motion dialogue between staff and students', Journal of Geography in Higher Education, 32(2), pp.303-320. https://doi.org/10.1080/03098260701514132.

Browne, M. N. and Keeley, S. M. (1998) Asking the right questions: a guide to critical thinking. $5^{\text {th }}$ edn. Upper Saddle River, NJ: Prentice Hall.

Clark, J., Gurney, L., Lawrence, S. Leece, R., Malouff, J., Masters, I., Reid, J., Tasker, I., Valenzuela, F. R. and Wilkes J. (2015) 'Embedding an institution-wide capacity building opportunity around transition pedagogy: first year teaching and learning network coordinators', The International Journal of the First Year of Higher Education, 6(1), pp.107-119. https://doi.org/10.5204/intifyhe.v6i1.268.

Devis-Rozental, C. (2018) Developing socio-emotional intelligence in higher education scholars. London: Palgrave. https://doi.org/10.1007/978-3-319-94036-6.

Devis-Rozental, C. and Barron, M. (2020) 'Mind the gap: supporting students to have a successful transition to university, it is everyone's responsibility', in Devis-Rozental, C. and Clarke, S. (eds.) Humanising higher education: a positive approach to wellbeing. Cham: Palgrave Macmillan, pp.83-106. https://doi.org/10.1007/978-3-030-57430-7 6.

Devis-Rozental, C. and Clarke, S. (eds.) (2020) Humanising higher education: a positive approach to wellbeing. Cham: Palgrave Macmillan. https://doi.org/10.1007/978-3-03057430-7. 
Devis-Rozental. C. and Farquharson, L. (2020) 'What influences students in their development of socio-emotional intelligence whilst at university?', Higher Education Pedagogies, 5(1), pp.294-309. https://doi.org/10.1080/23752696.2020.1820887.

Faber, K. and Fonseca, L. M. (2014) 'How sample size influences research outcomes', Dental Press J Orthod., 19(4), pp.27-29. https://doi.org/10.1590/2176-9451.19.4.027-029.ebo.

Fraser, W. J. and Killen, R. (2003) 'Factors influencing academic success or failure of firstyear and senior university students: do education students and lecturers perceive things differently?', South African Journal of Education, 23(4), pp. 254-260.

Greene, J. (2007) Mixed options in social inquiry. San Francisco: Jossey-Bass.

Groves, R. M., Fowler, F. J., Couper, M. P., Lepkowski, J. M., Singer, E. and Tourangeau, R. (2004) Survey Methodology. Hoboken: Wiley.

Hassel, S. and Ridout, N. (2018) 'An investigation of first-year students' and lecturers' expectations of university education', Frontiers in Psychology, 8, p.2218. https://doi.org/10.3389/fpsyg.2017.02218.

Houghton, A.-M. and Anderson, J. (2017) Embedding mental wellbeing in the curriculum: maximising success in higher education. London: HEA.

Jones, G. (2013) 'Supporting staff to be supporters', in Morgan, S. (ed.) Improving the student experience: a practical guide for universities and colleges. Abingdon: Routledge, pp.164-178. https://doi.org/10.4324/9780203817513-20.

Jones, S. (2018) 'Expectation vs experience: might transition gaps predict undergraduate students' outcome gaps?' Journal of Further and Higher Education, 42(7), pp.908-992. https://doi.org/10.1080/0309877X.2017.1323195. 
Mah, D.-K. and Ifenthaler, D. (2017) 'Academic staff perspectives on first-year students' academic competencies', Journal of Applied Research in Higher Education, 9(4), pp.630-640. https://doi.org/10.1108/JARHE-03-2017-0023.

Marshall, B., Cardon, P., Poddar, A. and Fontenot, R. (2013) 'Does sample size matter in qualitative research? a review of qualitative interviews in is research', Journal of Computer Information Systems, 54(1), pp.11-22.

https://doi.org/10.1080/08874417.2013.11645667.

Meehan, C. and Howells, K. (2018) 'What really matters to freshers? Evaluation of first year student experience of transition into university', Journal of Further and Higher Education, 42(7), pp.893-907. https://doi.org/10.1080/0309877X.2017.1323194.

Perry, R. P., Hladkyj, S., Pekrun, R. H. and Pelletier, S. T. (2001) 'Academic control and action control in the achievement of college students: a longitudinal field study', Journal of Educational Psychology, 93(4), pp.776-789. https://doi.org/10.1037/00220663.93.4.776.

Rozental-Devis, D. (2020) 'Humanising higher education by listening to the student voice', in Devis-Rozental, C. and Clarke, S. (eds.) Humanising higher education: a positive approach to wellbeing. Cham: Palgrave Macmillan, pp.65-82. https://doi.org/10.1007/978-3-030-57430-7 5.

Seldon, A. and Martin, A. (2018) The positive and mindful university. London: HEPI.

Tinto, V. (1999) 'Taking retention seriously: rethinking the first year of college', NACADA Journal, 19(2), pp.5-9. https://doi.org/10.12930/0271-9517-19.2.5.

van Rooij, E. (2018) Secondary school students' university readiness and their transition to university. Groningen: University of Groningen. 
Wojcieszek, L., Theaker, L., Ratcliff, M., MacPherson, L. and Boyd, J. (2014) 'Enhancing the first-year student experience through academic and professional staff collegiality', The International Journal of the First Year in Higher Education, 5(1), pp.143-151. https://doi.org/10.5204/intifyhe.v5i1.211.

Yorke, M. and Longden, B. (eds.) (2004) Retention and student success in higher education. Bodmin: Open University Press.

Yorke, M. and Longden, B. (2008) The first-year experience of HE in the UK. London: HEA.

\section{Author details}

Camila Devis-Rozental is Principal academic in socio-emotional intelligence and service excellence, Bournemouth University.

Susanne Clarke is Head of service excellence, Bournemouth University. 